\title{
Comparison of Kruis, Manning and Rome IV Criteria in Irritable Bowel Syndrome
}

\author{
Farah Naaz Kazi, Anurag Agarwal, Prashant Kanni \\ Department of Medical Gastroenterology, Vydehi Institute of Medical Sciences and Research Centre, Bangalore, Karnataka, India \\ Email address: \\ farahnaazkazi@gmail.com (F. N. Kazi), anuragarwal100@gmail.com (A. Agarwal), prashantyk@gmail.com (P. Kanni) \\ To cite this article: \\ Farah Naaz Kazi, Anurag Agarwal, Prashant Kanni. Comparison of Kruis, Manning and Rome IV Criteria in Irritable Bowel Syndrome. \\ International Journal of Gastroenterology. Vol. 4, No. 2, 2020, pp. 34-40. doi: 10.11648/j.ijg.20200402.12
}

Received: May 18, 2020; Accepted: June 20, 2020; Published: July 6, 2020

\begin{abstract}
Irritable Bowel Syndrome (IBS) is a functional disorder of the gastrointestinal tract characterized by chronic abdominal pain, cramping, constipation, and diarrhea. Manning criteria, Kruis criteria and Rome IV criteria have shown that certain symptoms derived from a 15-items questionnaire differentiated patients with Irritable Bowel Syndrome (IBS) from patients with organic diseases. The purpose of the study is to find out the reliability and discriminatory value of the Manning criteria, Kruis criteria and Rome IV criteria in the differentiation of Irritable Bowel Syndrome (IBS) from organic diseases and to find out if the three criteria could be combined. The study is a prospective cross-sectional analytical study of one hundred and thirty patients who presented with Diarrhea or Constipation to the Department of Medicine, Department of Surgery, Department of Medical Gastroenterology, Vydehi Institute of Medical Sciences and Research Centre, Bangalore between September- February, 2019-2020. After taking informed consent, patients were subjected to preformed questionnaire in the language best understood by them. Patient underwent diagnostic investigations like Complete blood count (CBC), Erythrocyte Sedimentation Rate (ESR), C- reactive protein, Serum albumin and Colonoscopy. A total of 130 patients were interviewed for the study. Manning criteria had the highest sensitivity (88\%) compared to Kruis criteria (81\%) and Rome IV criteria (80\%). Kruis criteria had the highest specificity (91\%) compared to Manning criteria (87\%) and Rome IV criteria (86\%). On combining the three criteria, while the sensitivity is $94.4 \%$, the specificity fell drastically to $58 \%$. Hence everything considered it is best to ply with the individual criteria for the diagnosis of irritable bowel syndrome.
\end{abstract}

Keywords: Irritable Bowel Syndrome (IBS), Kruis Criteria, Manning’s Criteria, Rome’s IV Criteria

\section{Introduction}

Irritable bowel syndrome (IBS) or irritable bowel disease (IBD) is also known as spastic colitis, mucus colitis, and nervous colon [1]. Irritable bowel syndrome (IBS) is a functional disorder that affects the GI tract and causes symptoms such as abdominal pain, cramping, constipation, and diarrhea. [2]

The etiology is poorly understood and many factors are involved.[3] Altered gastrointestinal motility, visceral hypersensitivity, post infectious reactivity, brain-gut interactions, alteration in fecal micro flora, bacterial overgrowth, food sensitivity, carbohydrate malabsorption, and intestinal inflammation all have been implicated in the pathogenesis of Irritable Bowel Syndrome (IBS) [3]. Serotonin is largely present in the enterochromaffin cells in the gut and is a major regulator of the peristaltic reflex and sensory relays in the gut [4]. There are two lines of evidence supporting the view that serotonin regulation is abnormal in Irritable bowel syndrome (IBS). The release of serotonin in plasma appears to be reduced in those with constipationpredominant Irritable bowel syndrome (IBS) (IBS-C) and increased in diarrhea-predominant Irritable bowel syndrome (IBS) (IBS-D) [5]. A defect in serotonin signaling was noted in both Irritable bowel syndrome (IBS) and ulcerative colitis, with a reduction in normal mucosal serotonin and serotonin transporter immune-reactivity in both diseases [6]. Psychological stress exacerbates gastrointestinal symptoms magnifying the severity of diarrhea, abdominal discomfort, and so on. Next, psychological and psychiatric co morbidity is often represented among Irritable bowel syndrome (IBS) patients. [7]

Irritable Bowel Syndrome (IBS) can be subdivided into those who tend to have IBS with diarrhea or IBS with 
constipation or IBS with mixed bowel habits or IBS unclassified $[8,9]$.

The Manning criteria were truly the first global Irritable Bowel Syndrome (IBS) diagnostic criteria to be introduced and have been the most extensively studied [10]. The four main symptoms included looser stools at the onset of pain, increased frequency of bowel movements after the onset of pain, relief of abdominal pain after a bowel movement, and abdominal distension. Two additional symptoms were found to be of increased prevalence in patients with Irritable Bowel Syndrome (IBS) (sensation of incomplete evacuation and fecal mucus).

In 1984, Kruis and colleagues reported on a similar set of symptoms used to define Irritable Bowel Syndrome (IBS): abdominal pain, bloating, and altered bowel function [11]. In contrast to the Manning criteria, the Kruis criteria placed a greater emphasis on symptom duration, and in fact suggested a two-year time duration. More importantly, the Kruis criteria highlighted the need to consider warning signs ("red flags") and also to exclude organic disease with a combination of a normal physical examination and basic laboratory studies (CBC and ESR).

Rome IV defined irritable bowel syndrome (IBS) as a functional bowel disorder in which recurrent abdominal pain is associated with defecation or a change in bowel habits. Disordered bowel habits are typically present (i.e., constipation, diarrhea or a mix of constipation and diarrhea), as are symptoms of abdominal bloating/distension. Symptom onset should occur at least 6 months prior to diagnosis and symptoms should be present during the last 3 months.

Functional gastrointestinal disorders (FGIDs) account for at least $40 \%$ of all referrals to gastroenterologists. Of the 33 recognized adult FGIDs, irritable bowel syndrome (IBS) is the most prevalent, with a worldwide prevalence estimated at $12 \%$ and in India at $4.2 \%$ [15]. Irritable Bowel Syndrome (IBS) is an important health care concern as it greatly affects patients' quality of life and imposes a significant economic burden to the health care system.

To overcome the obstacle of expensive and extensive workups, important attempts for positive diagnosis of Irritable Bowel Syndrome (IBS) from physical symptoms have been made by questionnaire surveys [10, 11]. Manning criteria, Kruis criteria and Rome IV criteria [10] have shown that certain symptoms derived from a 15-items questionnaire differentiated patients with Irritable Bowel Syndrome (IBS) from patients with organic diseases.

The purpose of the present study was to evaluate the reliability and discriminatory value of the Manning criteria, Kruis criteria and Rome IV criteria in the differentiation of Irritable Bowel Syndrome (IBS) from organic diseases.

\section{Objectives}

1. To find out the reliability and discriminatory value of the Manning criteria, Kruis criteria and Rome IV criteria in the differentiation of Irritable Bowel Syndrome (IBS) from organic diseases.
2. To establish if the three scorings (Manning criteria, Kruis criteria and Rome IV criteria) could be combined.

\section{Methodology}

This is a prospective cross-sectional analytical study done over a period of six months from September- February 20192020. The study was conducted in patients who present with Diarrhea or constipation to the Department of Medicine, Department of Surgery, Department of Medical Gastroenterology, Vydehi Institute of Medical Sciences and Research Centre, Bangalore. A total of 130 patients had taken part in this study. After explaining the details and purpose of the study to the participants and obtaining the oral informed consent, data was collected using preformed questionnaire in patient best understood language. Patients underwent routine investigations for this study. Complete blood count (CBC), Erythrocyte Sedimentation Rate (ESR), C- reactive protein, Serum albumin, Colonoscopy was done. Data was analyzed using Epi Info 7.1.5.

To calculate the specificity and sensitivity of the criteria, data was distributed according to the outcome of the diagnosis. True Positives are the patients with a positive score on IBS criteria set and no specific disease. Similarly, True Negatives are the patients with a negative score on IBS criteria set and have a specific disease. False Positives are the patients with positive score on IBS criteria set but have a specific disease. False Negatives are the patients with a negative score on IBS criteria set and have no specific disease. Sensitivity measures the proportion of actual positives that are correctly identified as such, that is, the percentage of study group who were correctly identified to have Irritable Bowel Syndrome (IBS). Specificity measures the proportion of actual negatives that are correctly identified as such, that is, the percentage of study group who were correctly identified to not have Irritable Bowel Syndrome (IBS). [16]

\subsection{Inclusion Criteria}

1. Patient presenting with Diarrhea / constipation for more than 6 months.

2. Age 18 to 80 years of either gender.

\subsection{Exclusion Criteria}

1. People with prior diagnosed gastrointestinal disorders.

2. Patients who are in shock, ARDS, ventilator support.

3. Patients who have already participated in the project and have come for follow-up.

4. Red flags, increased inflammatory markers, hemoglobin less than $7 \mathrm{~g} \%$.

5. Patients with microscopic/ lymphocytic/ collagenous colitis, inflammatory bowel disease, celiac disease, visceral cancer, chronic infectious disease, immunodeficiency, uncontrolled thyroid disease.

6. Patients with history of liver disease or elevated AST/ ALT $>2$ times the upper limit of normal levels. 
7. Patients with prior radiation therapy of the abdomen or abdominal surgeries with the exception of appendicectomy or cholecystectomy $>6$ months prior to study initiation.

8. Ingestion of any over the counter drugs or herbal medications which can affect GI transit or study interpretation (example: opioids, narcotics, anticholinergic, norepinephrine reuptake inhibitors, non- steroidal antiinflammatory drugs, COX-2 inhibitors, bile acid sequestrants) within 6 months of study initiation.

9. Any females who are pregnant or trying to become pregnant or breast feeding.

10. Antibiotic usage within 3 months prior to study participation.

11. Prebiotic or probiotic usage 2 weeks prior to study initiation.

12. Use of tobacco products within the past 6 months.

\section{Results}

A total of 130 patients were interviewed for the study. All the patients were asked to answer the three questionnaires, i.e., Kruis, Manning and Rome IV. Of the 130 patients, 84 patients were male and 46 patients were female, suggesting male predominance. Most of the patients were between the ages of 41-60 years. 43 patients presented with diarrhoea as their chief complaints, 77 patients presented with constipation as chief complaints and 10 patients with alternating diarrhea and constipation.

According to Kruis Criteria, 77 patients were diagnosed to have Irritable Bowel Syndrome and 53 patients were not diagnosed as Irritable Bowel Syndrome. Of the 130 patients interviewed, 73 patients were found to be true positive and 4 patients were false negative, 17 patients were found to be false positive and 36 patients were found to be true negative. Thus, Kruis criteria showed a sensitivity of $81 \%$ and a specificity of $91 \%$.

According to Manning Criteria, 83 patients were diagnosed to have Irritable Bowel Syndrome and 47 patients were not diagnosed as Irritable Bowel Syndrome. Of the 130 patients interviewed, 80 patients were found to be true positive and 3 patients were false negative, 21 patients were found to be false positive and 26 patients were found to be true negative. Thus, Manning criteria showed a sensitivity of $88 \%$ and a specificity of $87 \%$.

According to Rome IV Criteria, 74 patients were diagnosed to have Irritable Bowel Syndrome and 56 patients were not diagnosed as Irritable Bowel Syndrome. Of the 130 patients interviewed, 68 patients were found to be true positive and 6 patients were false negative, 18 patients were found to be false positive and 38 patients were found to be true negative. Thus, Rome IV criteria showed a sensitivity of $80 \%$ and a specificity of $86 \%$.

Thus, Manning criteria has the highest sensitivity (88\%) compared to Kruis criteria (81\%) and Rome IV criteria (80\%). Whereas, Kruis criteria has the highest specificity (91\%) compared to Manning criteria (87\%) and Rome IV criteria $(86 \%)$.
The three criteria (Kruis criteria, Manning criteria and Rome IV criteria) were then combined to check their sensitivity and specificity for the diagnosis of Irritable Bowel Syndrome (IBS).

Manning criteria and Rome IV criteria were combined. The combined Manning criteria and Rome IV criteria showed a sensitivity of $70 \%$ and a specificity of $98 \%$ with a $p$ value of 0.026 (significant).

Rome IV criteria and Kruis criteria were combined. The combined Rome IV criteria and Kruis criteria showed a sensitivity of $64.8 \%$ and a specificity of $98.7 \%$ with a $p$ value of 0.141 (insignificant).

Manning criteria and Kruis criteria were combined. The combined Manning criteria and Kruis criteria showed a sensitivity of $71 \%$ and a specificity of $98 \%$ with a $p$ value of 0.344 (insignificant).

Manning criteria, Kruis criteria and Rome IV criteria were combined. Thus, the combined Manning criteria, Kruis criteria and Rome IV criteria showed a sensitivity of $58 \%$ and a specificity of $88 \%$.

Amongst all the combined criteria, Kruis criteria and Manning criteria showed highest sensitivity (71\%) towards diagnosis of Irritable Bowel Syndrome (IBS). Kruis criteria and Rome IV criteria showed highest specificity (98.7\%) towards the diagnosis of Irritable Bowel Syndrome (IBS).

Table 1. Data showing kruis criteria and their outcome (using both questinnaire and relevant investigation).

\begin{tabular}{lll}
\hline & DISEASE PRESENT & DISEASE ABSENT \\
\hline POSITIVE & 73 & 17 \\
NEGATIVE & 4 & 36 \\
TOTAL & 77 & 53 \\
\hline
\end{tabular}

Table 2. Data showing manning criteria and their outcome (using both questinnaire and relevant investigation).

\begin{tabular}{lll}
\hline & DISEASE PRESENT & DISEASE ABSENT \\
\hline POSITIVE & 80 & 21 \\
NEGATIVE & 3 & 26 \\
TOTAL & 83 & 47 \\
\hline
\end{tabular}

Table 3. Data showing rome iv criteria and their outcome (using both questinnaire and relevant investigation).

\begin{tabular}{lll}
\hline & DISEASE PRESENT & DISEASE ABSENT \\
\hline POSITIVE & 68 & 18 \\
NEGATIVE & 6 & 38 \\
TOTAL & 74 & 56 \\
\hline
\end{tabular}

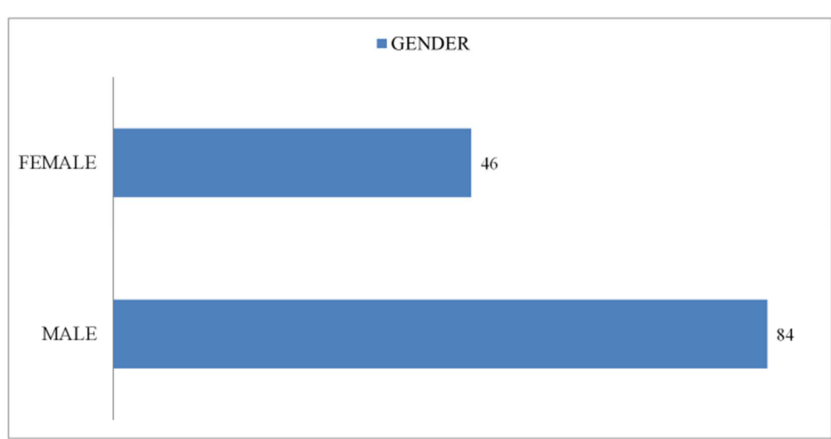

Figure 1. Depiciting the gender distribution in the study. 


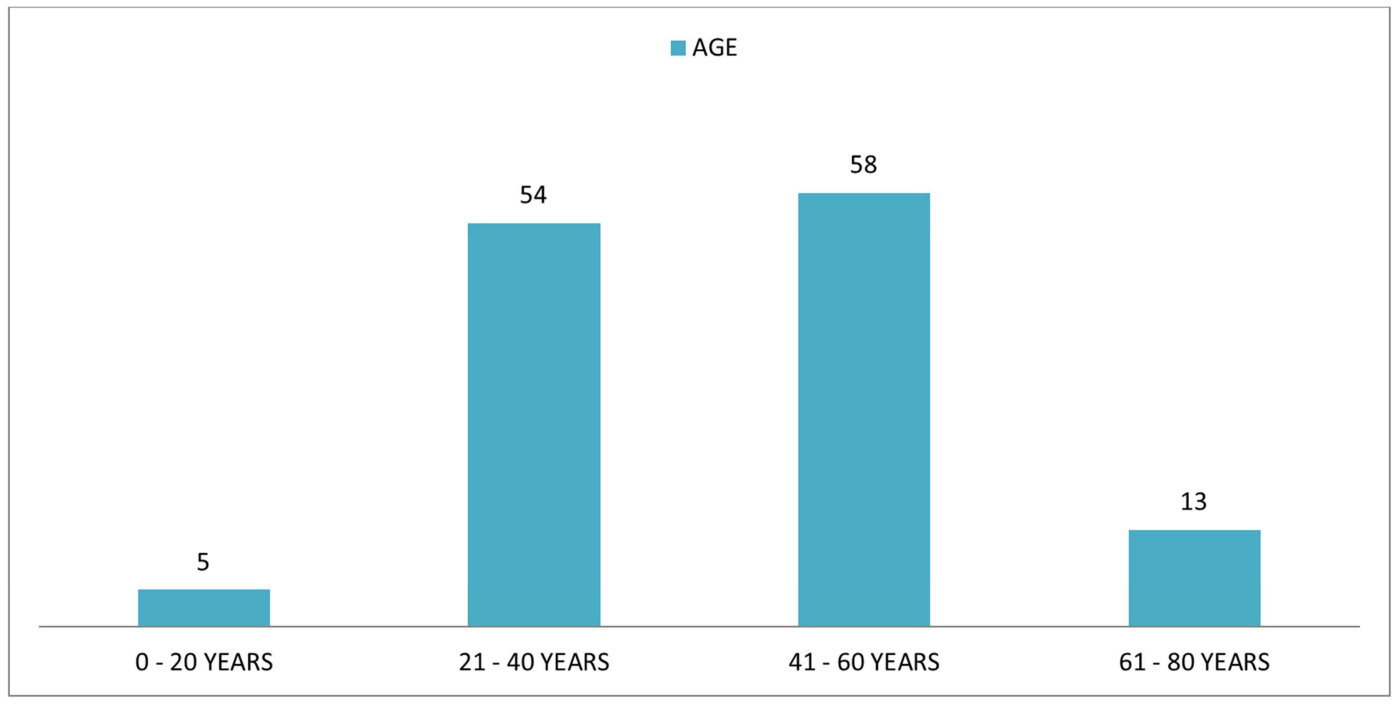

Figure 2. Depicting distribution of age group of the patients in the study group.

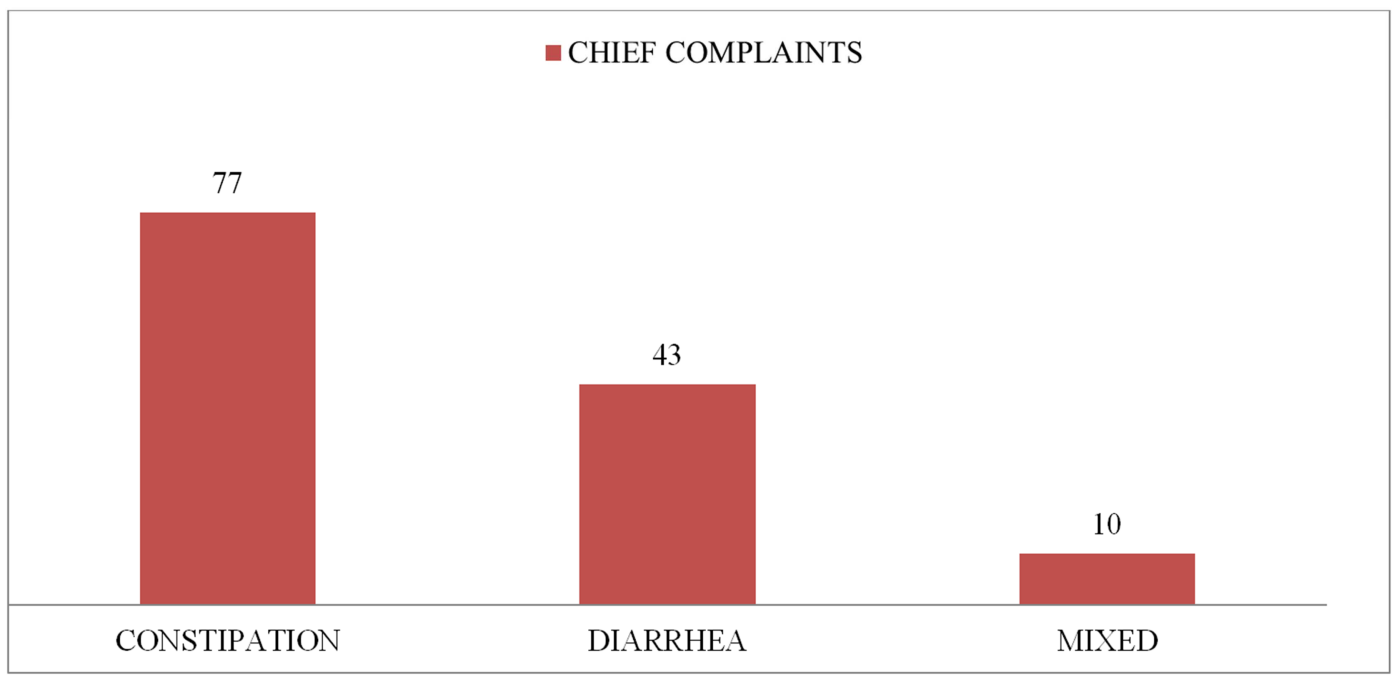

Figure 3. Depiciting the distribution of chief complaints in the study group.

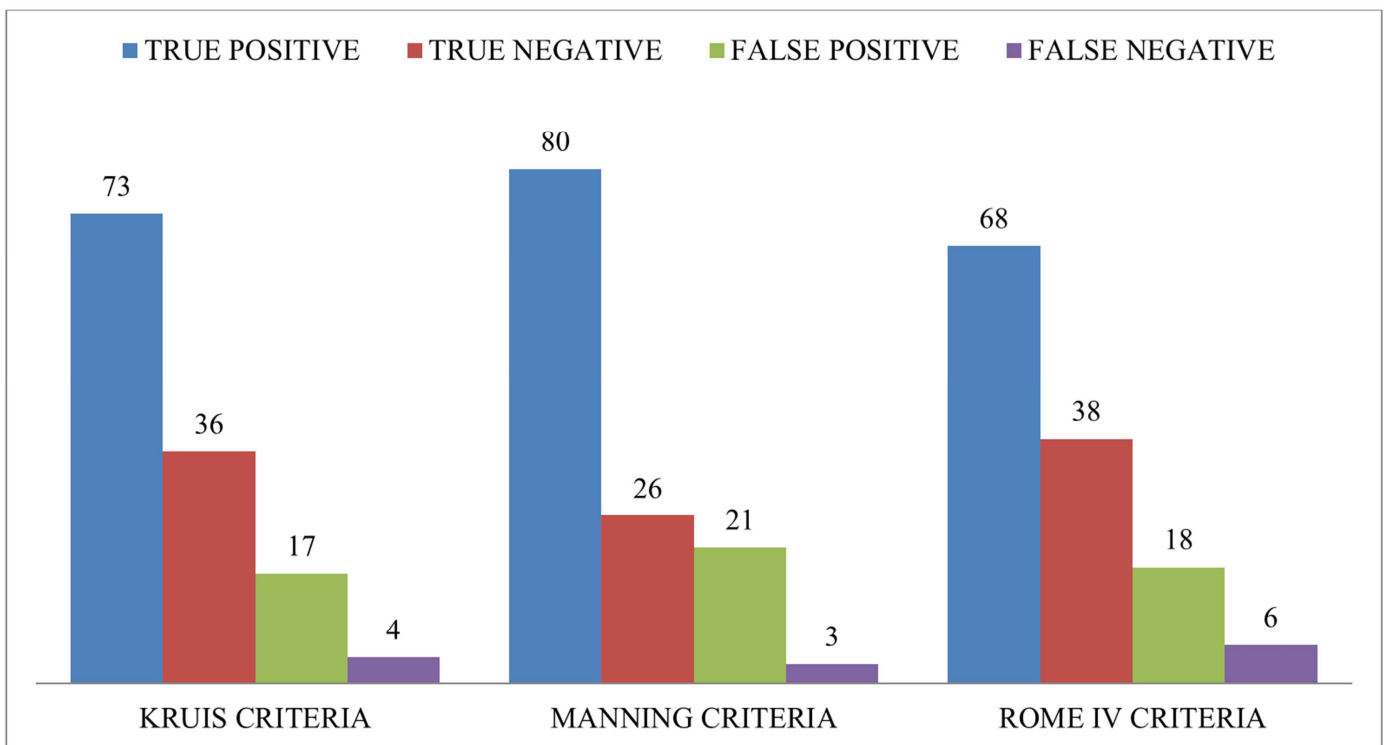

Figure 4. Depiciting the diagnosis distribution according to the three criteria (rome iv criteria, manning criteria, and kruis criteria) used in the study. 


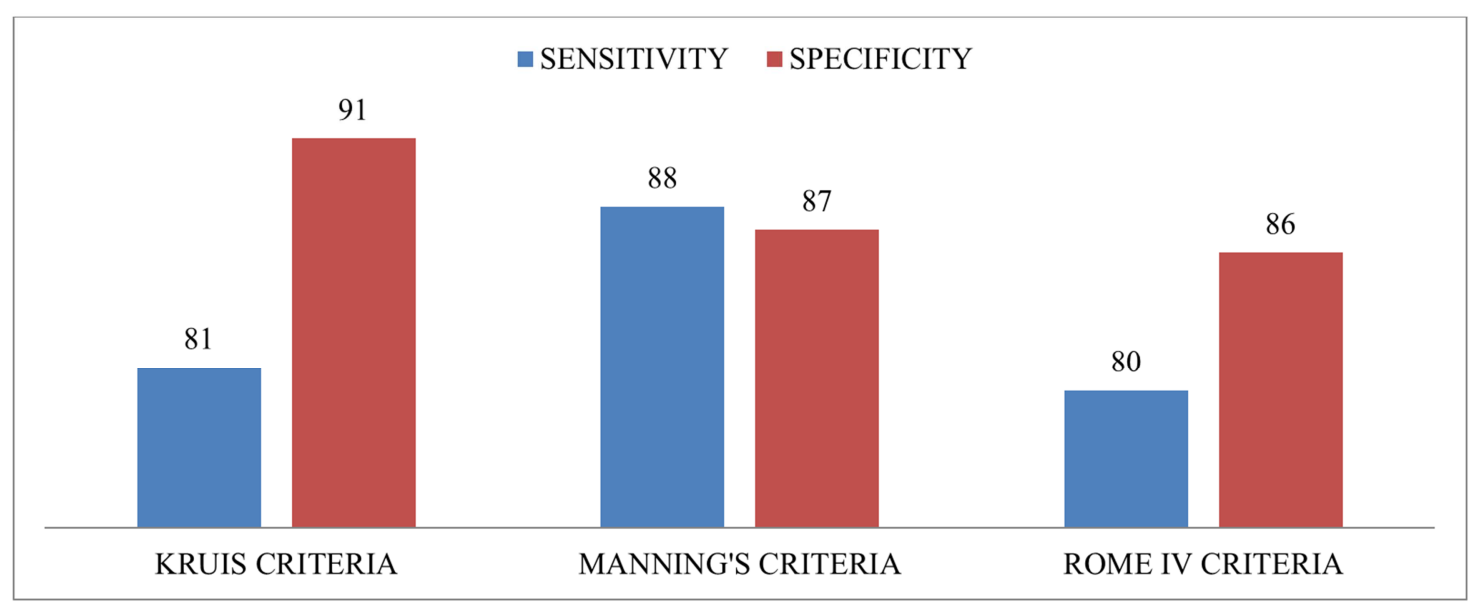

Figure 5. Depicting the distribution of sensitivity and specificity with respective to different criteria used in the study.

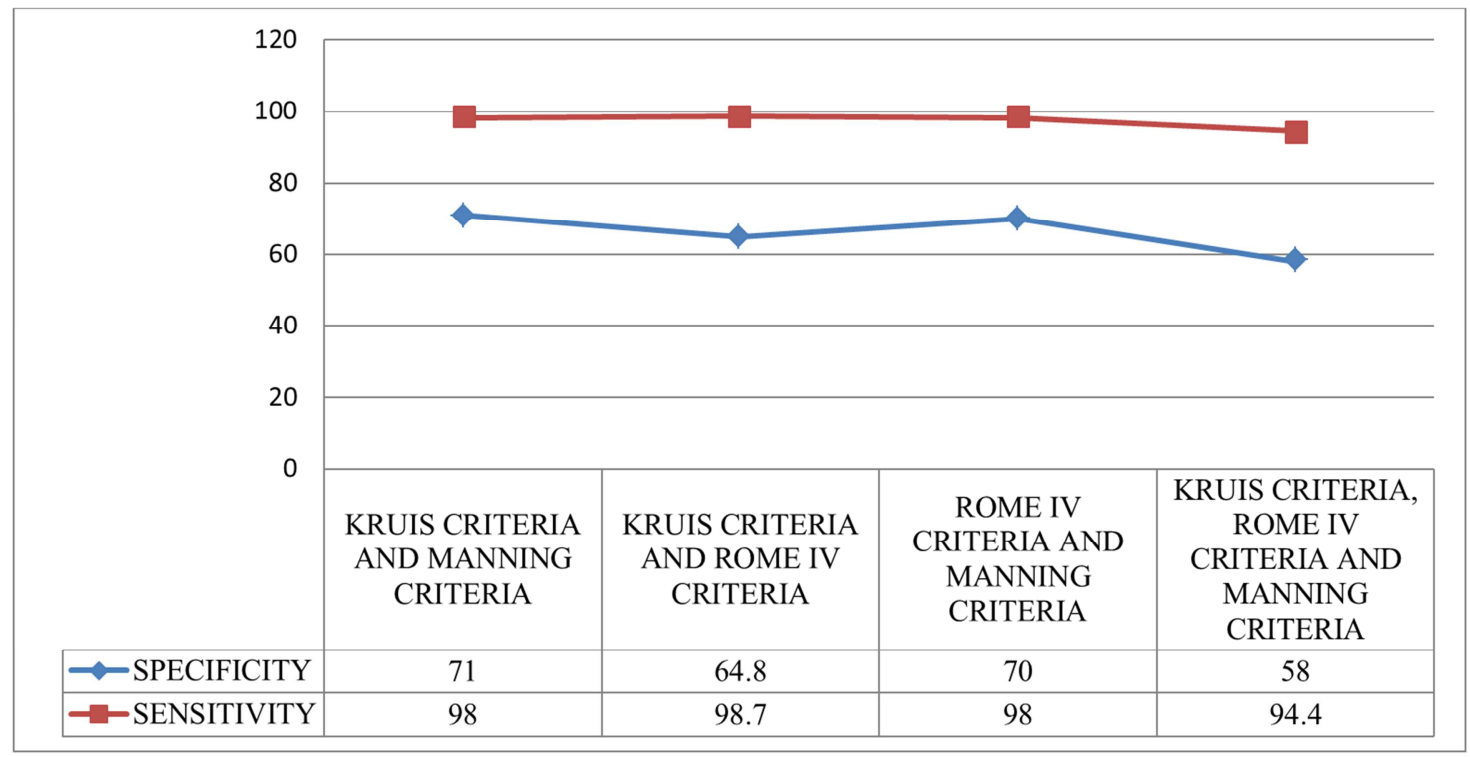

Figure 6. Depiciting the specificity and sensitivity distribution according to the three criteria (rome iv criteria, manning criteria, and kruis criteria) used in the study.

\section{Discussion}

The performance of symptom-based IBS criteria in the exclusion of organic diseases was highly variable, both across criteria sets and between studies evaluating the same IBS criteria. With regard to the exclusion of specific GI diseases, our results showed that patients who fulfilled IBS criteria have a lower risk for specific GI diseases than those not fulfilling the criteria. The absence of an alarm symptom does not seem to be helpful in confirming an IBS diagnosis, while presence of infrequent bowel movements does.

in our study, patients were between age group of 16 to 80 years with mean age of patients being 56 which is similar to the study by Dogan and Unal [13]. However, male:female ratio found in our study was 1.8:1 while that done by Dogan and Unal for IBS was $0.7: 1$ and for OGD was 1.5:1. This disparity in the study is due to the fact that females in india often do not come out very often to seek medical attention. Hence the number of cases reported are also less.
The questionnaire study of manning et al [10] showed that the more the following symptoms were reported, the more likely was the diagnosis of IBS: abdominal distension, relief of pain with bowel habits, loose and more frequent stools with onset of pain, presence of mucus in stools, and incomplete bowel evacuation. Sensitivity of presence of three or more symptoms of Manning's criteria discriminating irritable bowel syndrome from all other groups was $66.1 \%$. Manning's criteria discriminated irritable bowel syndrome from organic diseases of colon with specificity and positive predictive value of $70 \%$ and $82.6 \%$. Among subgroups of the IBS groups, the pain-predominant subgroup showed higher score and frequency than the painless subgroup. With the above results, we expect that the Manning criteria is useful as a backup tool for the diagnosis of IBS and more specific in pain-predominant IBS subgroup. Among the individual symptoms, 'more frequent stools at pain onset' and 'pain relief with defecation' were significantly more frequent in IBS group compared with organic gastrointestinal disease group. These two symptoms were in concordance with 
'irritable bowel factors'), which were relief of pain with defecation, looser stools with pain onset, more frequent stools with pain, and gastrointestinal reactions to eating. These results proved to be similar to the results of the previous study.

Kruis et al [11] also found that the following symptoms when combined, correlated highly with IBS, abdominal pain, flatus and irregular bowel habits. three of four studies that examined the accuracy of the Kruis symptom score suggested it provides an excellent positive predictive value with a high sensitivity (64\%) and specificity (99\%). Severe organic diseases were very well discriminated by the score at a level of 44 points. In particular, the score of patients with a carcinoma was far below this level. According to these findings, it seems justified to treat patients with $>44$ points as IBS and to withhold further diagnostic steps.

Manning and kruis $[10,11]$ is associated with high false positive. They showed to have a sensitivity of $81 \%$ and specificity of $91 \%$ when combined together. But, these are best available diagnostic criteria.

Extensive study has been done on diagnosis of IBS by rome III. However, no research has been done on Rome IV criteria. Also no comparative study of Romes criteria has been done with either Manning's or Kruis.

Table 4. Data comparing the results of the different criteria in previous report.

\begin{tabular}{|c|c|c|c|c|c|c|}
\hline Study & True positive & False positive & False negative & True negative & Sensitivity & Specificity \\
\hline MANNING [10] & 29 & 10 & 3 & 23 & $91 \%(75-98 \%)$ & $70 \%(51-84 \%)$ \\
\hline KRUIS [11] & 69 & 2 & 39 & 207 & $64 \%(54-73 \%)$ & $99 \%(97-100 \%)$ \\
\hline ROME III [15] & 251 & 304 & 113 & 1179 & $68.8 \%$ & $79.5 \%$ \\
\hline DOGAN AND UNAL [13] & 134 & 16 & 31 & 166 & $81 \%(74-87 \%)$ & $91 \%(86-95 \%)$ \\
\hline
\end{tabular}

However there are some limitations to the questionnaire. The questionnaire being an elaborate one is quite time consuming and cumbersome on the part of the physician and large variability of subjectively complained symptoms and their tolerance, and different sociocultural or psychological situation for health care seeking behavior on the part of the patient makes it difficult to keep a long term follow-up. Also, $100 \%$ specificity is not possible as irritable bowel syndrome symptoms are quite common in the general population and the predictive value of the three criteria in our study is based on the prevalence of the disease in the population being assessed. However the patients were co-operative to the study and this has lead to an ideal result.

Our study indicates that the Manning, Kruis and Romes criteria are reasonably specific and of diagnostic value. Positive result with the three scoring system is consistent with the diagnosis of IBS. Development of better diagnostic criteria, with improved accuracy by some modification based on further studies, should prevent unnecessary, extensive investigations for the diagnosis of IBS.

\section{Conclusion}

A considerable number of patients who visit the gastroenterology department have functional disorders of the gastrointestinal tract. Therefore, it becomes of utmost importance on the part of the physicians to rule out all the other organic disorders before making a diagnosis of irritable bowel syndrome. Its multifactorial pathogenesis and difference in perception of symptoms by patients makes detection of irritable bowel syndrome a real challenge. The massive expenditure on investigations are not always in the patient's favour. However, with comprehensive questioning of patients based on the Kruis', Manning's and Rome IV criteria, the apt diagnosis and minimizing the redundant health care costs can be achieved.
From the study, we conclude that Manning's criteria has the highest sensitivity while Kruis' criteria has the highest specificity. On the other hand, the specificity and sensitivity of Rome's IV criteria is intermedial to that of Kruis' and Manning's and hence is being used widely for diagnosing Irritable bowel Syndrome lately. However, on combining the three criteria, while the sensitivity is realistic, the specificity fell drastically. Therefore, the amalgamation of the criteria does not lead to the explicit confirmation of irritable bowel syndrome and proves to be tedious for the physician and the patients. Hence everything considered it is best to ply with the individual criteria for the diagnosis of irritable bowel syndrome.

\section{Conflict of Interest}

The authors declare that they have no competing interests.

\section{References}

[1] Brandt LJ, Chey WD, Foxx-Orenstein AE, et al. An evidencebased systematic review on the management of irritable bowel syndrome. American Journal of Gastroenterology. 2009; 104 (Suppl 1): S1-S35.

[2] Boyce PM, Koloski NA, Talley NJ. Irritable bowel syndrome according to varying diagnostic criteria: are the new Rome II criteria unnecessarily restrictive for research and practice. Am J Gastroenterol. 2000 Nov; 95 (11): 3176-83.

[3] Occhipinti K, Smith JW. Irritable bowel syndrome: a review and update. Clin Colon Rectal Surg. 2012; 25: 46-52.

[4] Talley NJ. Serotoninergic neuroenteric modulators. Lancet. 2001; 358: 2061-2068.

[5] Dunlop SP, Coleman NS, Blackshaw E, Perkins AC, Singh G, Marsden CA, Spiller RC. Abnormalities of 5hydroxytryptamine metabolism in irritable bowel syndrome. Clin Gastroenterol Hepatol. 2005; 3: 349-357. 
[6] Coates MD, Mahoney CR, Linden DR, Sampson JE, Chen J, Blaszyk H, Crowell MD, Sharkey KA, Gershon MD, Mawe $\mathrm{GM}$, et al. Molecular defects in mucosal serotonin content and decreased serotonin reuptake transporter in ulcerative colitis and irritable bowel syndrome. Gastroenterology. 2004; 126: $1657-1664$.

[7] Drossman DA, Camilleri M, Mayer EA, Whitehead WE. AGA technical review on irritable bowel syndrome. Gastroenterology. 2002; 123: 2108-2131.

[8] Drossman DA, Corrazziari E, Delvaux M, Spiller R, Talley NJ, Thompson WG. Rome III: The Functional Gastrointestinal Disorders. Gastroenterology 2006; 130: 1377-1390.

[9] Talley NJ, Spiller R. Irritable bowel syndrome: a little understood organic bowel disease? Lancet. 2002; 360: 555564.

[10] Manning A. P., Thompson W. G., Heaton K. W., Morris A. F. Towards positive diagnosis of the irritable bowel. Br. Med. J. 1978; 2: 653-654.

[11] Kruis W., Thieme C., Weinzierl M., Schüssler P., Holl J., Paulus W. A diagnostic score for the irritable bowel syndrome. Its value in the exclusion of organic disease. Gastroenterology August 1984; 87 (1): 1-7.
[12] Homes KM, Salter RH. Irritable bowel syndrome-a safe diagnosis? Br Med J. Manning Criteria in Irritable Bowel Syndrome: Its Diagnostic Significance. Korean J Intern Med. 1993 Jan; 8 (1): 34-39.

[13] Doğan UB, Unal S. Kruis scoring system and Manning's criteria in diagnosis of irritable bowel syndrome: is it better to use combined? Acta Gastroenterol Belg. 1996 Oct-Dec; 59 (4): 225-8.

[14] Ford A. C., Bercik P., Morgan D. G., Bolino C., PintosSanchez M. I., Moayyedi P. Validation of the Rome III criteria for the diagnosis of irritable bowel syndrome in secondarycare. Gastroenterology. 2013; 145: 1262-1270.

[15] Andrew seng boon chua. Prevalence of irritable bowel syndrome in northern india. J neurogastroenterol motil. 2011 Jan; 17 (1): 6-8.

[16] P. Jellema, D. A. W. M. Van Der Windt, F. G. Schellevis, H. E. Van Der Horst. Systematic review: accuracy of symptombased criteria for diagnosis of irritable bowel syndrome in primary care. 2009 September; 30 (7): 695-706. 